\title{
LA EXPERIENCIA DE INTERCAMBIO ESTUDIANTIL EN EL EXTRANJERO: Análisis de las percepciones de chilenos que en su adolescencia participaron en programas de youth for understanding
}

\author{
MARÍA CECILIA FIOCCHI* \\ HUGO ROJAS $^{* *}$
}

\begin{abstract}
RESUMEN
En este documento se analiza la experiencia de intercambio estudiantil de seis chilenos que viajaron al extranjero durante su adolescencia. Transcurridos varios años desde su retorno al país, se explica cómo los participantes evalúan la experiencia de intercambio que vivieron y las principales lecciones aprendidas. Se concluye que los programas de intercambio contribuyen a un conjunto de cambios positivos en el sujeto y que se aprecian en: una mayor valoración de la diversidad cultural, el aumento de la confianza en sí mismo, el desarrollo de pensamiento propio e independiente, mejores habilidades comunicacionales y de interacción social, y una mayor capacidad adaptativa frente a nuevas circunstancias y desafíos.
\end{abstract}

\section{PALABRAS CLAVE: INTERCAMBIO ESTUDIANTIL, ADAPTACIÓN CULTURAL, DIVERSIDAD CULTURAL, YOUTH FOR UNDERSTANDING}

* Licenciada en Gobierno y Relaciones Internacionales (Universidad Argentina de la Empresa), Diplomada en Integración Regional (Instituto de Estudios Internacionales, Universidad de Chile), estudiante de intercambio de YFU Argentina en Holanda (2007-08). Ayudante de Investigación en la Universidad Alberto Hurtado y Pasante en YFU Chile (2014). Agradece a Carlos Costa, Kathleen Neidhardt, Doug Soffer, Joshua Beatty, Luis Pardo y Claudia Martins, y a cada una de las personas que aceptaron ser entrevistadas para este proyecto, tanto en su etapa de formulación (plan piloto) como en su desarrollo posterior.

E-Mail:mfiocchi@uade.edu.ar.

** Profesor de Sociología del Derecho, Facultad de Derecho de la Universidad Alberto Hurtado. Candidato a Doctor en Sociología (University of Oxford), Master en Derecho, Antropología y Sociedad (London School of Economics \& Political Science), Magíster en Gerencia y Políticas Públicas (Universidad Adolfo Ibáñez), estudiante de intercambio de YFU Chile en Ohio, Estados Unidos (1988-89).

E-Mail: hurojas@uahurtado.cl. Una versión preliminar de este texto fue presentada en YFU Chile, el 27 de septiembre de 2014. Todos los errores son de responsabilidad de los autores. 


\title{
A EXPERIÊNCIA DO INTERCÂMBIO ESTUDANTIL NO ESTRANGEIRO: ANÁLISE DAS PERCEPÇÕES DOS CHILENOS QUE EM SUA ADOLESCÊNCIA PARTICIPARAM EM PROGRAMAS DE YOUTH FOR UNDERSTANDING
}

\begin{abstract}
RESUMO
Neste documento é analisada a experiência do intercâmbio estudantil de seis chilenos que viajaram para o estrangeiro durante a adolescência. Depois de vários anos de sua volta ao país, é explicada a maneira como os participantes avaliam a experiência de intercâmbio que viveram e as principais lesões aprendidas. É possível concluir que os programas de intercâmbio contribuem para um conjunto de mudanças positivas no sujeito como por exemplo: maior valoração da diversidade cultural, o aumento da confiança em si mesmo, o desenvolvimento do pensamento próprio e independente, melhores habilidades comunicacionais e de interação social e uma maior capacidade adaptativa para afrontar novas circunstancias e desafios.
\end{abstract}

\section{PALAVRAS CHAVE: INTERCÂMBIO ESTUDANTIL, ADAPTAÇÃO CULTURAL, DIVERSIDADE CULTURAL, YOUTH FOR UNDERSTANDING}

\section{THE STUDENT EXCHANGE ABROAD EXPERIENCE: AN ANALYSIS OF THE PERCEPTIONS OF CHILEAN PEOPLE WHO PARTICIPATED IN YOUTH FOR UNDERSTANDING PROGRAMS IN THEIR ADOLESCENCE}

\begin{abstract}
The present article analyzes the experiences of six Chilean exchange students who traveled abroad during their adolescence. After several years since their return to the country, the participants evaluate their exchange experience and the main lessons learned. We can conclude that exchange programs encourage the development of a series of positive changes such as an increased appreciation of cultural diversity, an increased self-confidence, the development of independent thinking, enhanced communication and social interaction skills, and more adaptability to face new circumstances and challenges.
\end{abstract}

KEY WORDS: STUDENT EXCHANGE, CULTURAL ADAPTATION, CULTURAL DIVERSITY, YOUTH FOR UNDERSTANDING 


\section{INTRODUCCIÓN}

EN ESTE DOCUMENTO SE explican algunos de los efectos en el largo plazo de la experiencia de intercambio estudiantil en tres generaciones diferentes de chilenos que han participado en programas de intercambio estudiantil. Gracias a los programas de intercambio, un adolescente se traslada a vivir durante varios meses a otro país, integrándose a una familia anfitriona y asistiendo regularmente a clases en un colegio. Tales programas se fundamentan en la importancia que reviste para la paz y cooperación mundial el hecho que personas de distintas culturas interactúen y comprendan por qué las sociedades viven y piensan diferente. Durante la experiencia se espera que los participantes desarrollen un conjunto de habilidades, competencias, actitudes y conocimientos, para así intervenir de mejor manera en un mundo cada vez más globalizado. Los adolescentes que se radican en el extranjero por un tiempo y que adquieren aprendizajes que los diferencian del resto (Reyes et al., 2013), pueden asumir en el futuro el rol de agentes de cambio social e innovación (Caumont, 2014). Es evidente que la experiencia de intercambio estudiantil puede incidir en las distintas dimensiones del desarrollo humano - cognitivo, social, afectivo, moral y físico - de los participantes. Por otra parte, la experiencia juvenil en la sociedad actual está cada vez más asociada a nuevos contextos y nuevas modalidades de expresión de identidad (Sepúlveda, 2013:14). El sentido de esta investigación es contribuir a una mejor comprensión de la evaluación que hacen los ex alumnos chilenos de programas de intercambio de la experiencia de haber residido en otro país durante un año.

En la actualidad se ha hecho cada vez más evidente la relevancia de alentar y apoyar la internacionalización de la educación a través de programas de intercambio. A fin de incrementar la comprensión y tolerancia entre distintas culturas, existe consenso en la comunidad internacional de las ventajas de los intercambios socio-culturales que se logran a partir de este tipo de iniciativas (UNESCO, 1998). Dada la necesidad actual impuesta por la globalización de ser ciudadanos con capacidades de comprender múltiples realidades, culturas y situaciones, cada año es mayor la cantidad de jóvenes que optan por vivir o estudiar un año en el exterior (Daly, 2002). Los jóvenes que realizan una experiencia de intercambio persiguen diversos objetivos, entre los cuales cabe destacar: aprender otro idioma, viajar, conocer personas diferentes, alejarse por un tiempo de su familia, comunidad o país de origen, salir de la rutina diaria y aprender realidades nuevas, etcétera. 
(IJAB, 2005). Dado que muchos de estos intercambios ocurren en la educación secundaria, no todos los participantes toman consciencia de los aprendizajes adquiridos y de cómo dicha experiencia incidirá en sus identidades y vocaciones tras regresar al país de origen. Dado que los sujetos adquieren las identidades sociales que su propio desarrollo sociocultural les ofrezca (Castro, 2007:14), la identidad de los jóvenes que viajan de intercambio se ve inevitablemente modificada a partir de la experiencia en el extranjero. La literatura especializada ha dado cuenta de los beneficios de convivir durante varios meses en una realidad alterna a la propia (Hansel, 1986; Nunan, 2006; Thomas, 2006). Algunos de los aprendizajes que suelen ser desarrollados por los participantes en programas de intercambio estudiantil son: desarrollo de habilidades interpersonales, disminución de prejuicios y estereotipos, interés por fenómenos globales, capacidad de comparar distintas realidades o fenómenos sociales, mayor orientación hacia lo internacional por sobre lo local, etcétera (Bachner y Zeutschel, 1994; Detweiler, 1984; Wilson, 1985).

\section{YOUTH FOR UNDERSTANDING CHILE}

Con la finalidad de contribuir al restablecimiento de confianzas entre países enemigos durante la Segunda Guerra Mundial, 75 alemanes tuvieron la oportunidad de convivir durante un año con familias del estado de Michigan, Estados Unidos, en 1951. Rachel Andresen, directora ejecutiva del Ann Arbor/Washtenaw Council of Churches, tuvo un rol fundamental en la coordinación de las familias anfitrionas en Michigan. Dado que esa experiencia fue evaluada positivamente, en 1952 se creó oficialmente la organización Youth For Understanding, por sus siglas en inglés, YFU. El programa de intercambio se expandió en la década del 50 a otros países europeos y asiáticos. Actualmente YFU realiza intercambios estudiantiles en más de 55 países, de todos los continentes, dando apoyo a más de cuatro mil jóvenes cada año. YFU se define como una «organización educativa, sin fines de lucro, sin filiación política, religiosa, ni racial, dedicada a promover el entendimiento intercultural, el respeto mutuo y la responsabilidad social por medio de intercambios educacionales para jóvenes, familias y comunidades» (YFU, 2012). Las oficinas nacionales de YFU se encargan de: a) reclutar estudiantes nacionales, b) buscar familias anfitrionas, y c) acompañar y apoyar a los estudiantes y familias durante sus experiencias. Para poder llevar a cabo las distintas actividades de 
selección, capacitación, coordinación, acompañamiento, difusión, evaluación, etcétera, los voluntarios —en su mayoría ex participantes de los programas - juegan un rol trascendental en las distintas oficinas regionales.

En 1963, YFU comenzó a operar en Chile. En estos cincuenta años han participado 7.955 personas en sus programas de intercambio: 6.416 chilenos han viajado al exterior y 1.539 extranjeros han residido en Chile. El 91\% de los chilenos ha viajado a Estados Unidos, y el 9\% restante a otros países. Cabe mencionar que entre 1963 y 1990, los chilenos que participaron en los programas de YFU solo pudieron viajar a Estados Unidos. A partir de 1990, los adolescentes pudieron optar por Alemania, y en las últimas dos décadas se ha diversificado la cantidad de países, llegando en la actualidad a 31. Por ejemplo, actualmente los participantes chilenos de YFU residen en doce países. Por su parte, el $48 \%$ de los estudiantes de intercambio en Chile ha provenido de Estados Unidos, mientras que el 52\% restante lo ha hecho desde otros países. Con todo, la tendencia es hacia una mayor diversificación de países de intercambio, tanto de origen como de destino. El Gráfico 1 muestra la cantidad de chilenos que han participado en programas de YFU en el extranjero en los últimos cincuenta años. El Gráfico 2 muestra la cantidad de extranjeros que han residido en Chile con ocasión de los programas de YFU.

GRÁFICO 1: PARTICIPANTES CHILENOS EN PROGRAMAS DE YFU EN EL EXTRANJERO

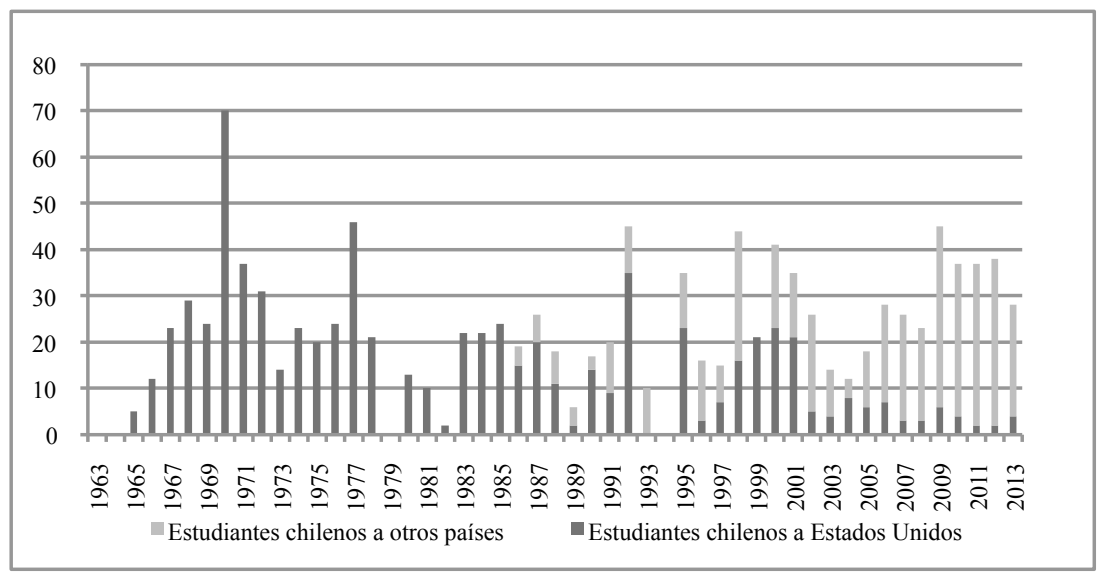

Fuente: elaboración propia a partir de datos proporcionados por YFU Chile $(\mathrm{N}=6.416)$. 
GRÁFICO 2: PARTICIPANTES EXTRANJEROS EN PROGRAMAS DE YFU EN CHILE

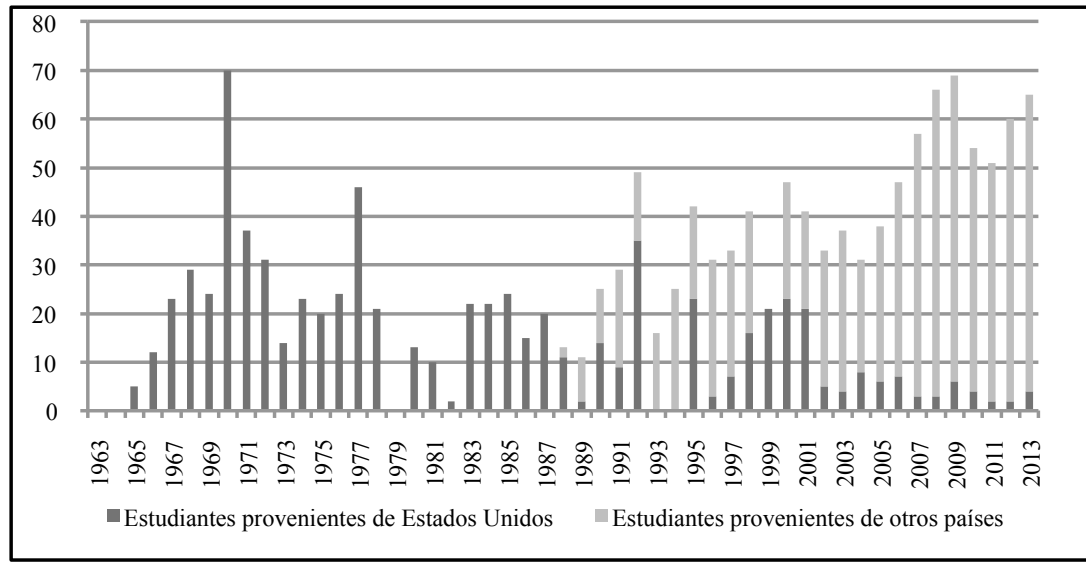

Fuente: elaboración propia a partir de datos proporcionados por YFU Chile $(\mathrm{N}=1.539)$.

YFU Chile ofrece dos tipos de programas: convencionales y especiales. Dentro de los convencionales, el estudiante puede optar por viajar entre un semestre o un año académico a otro país. A modo de ejemplo, en el último año 53 chilenos optaron por el programa de intercambio de un año de duración y 21 por un semestre (2013-14). Los programas especiales, además de la convivencia con familias anfitrionas y asistencia al colegio, incluyen actividades específicas relacionadas con arte, música, moda, inglés, cine, naturaleza, gastronomía o básquetbol. El costo que tiene para un chileno viajar por un año al extranjero varía de 190 a 356 unidades de fomento, dependiendo del lugar de destino. Dicho monto incluye los gastos en pasajes aéreos de ida y regreso, visa, seguro de salud, clases de idioma, orientaciones y gastos operativos de YFU. Los padres además deben considerar un monto mensual para los gastos personales del estudiante, recomendado en US $\$ 250$ dólares (o equivalente). El número de becas disponible es reducido, por lo que la inmensa mayoría de los participantes chilenos proviene de hogares de clase media alta o alta. Los requisitos generales para participar en los programas de intercambio son los siguientes:

- Tener entre 15 y 18 años de edad.

- Tener un dominio al menos básico del idioma inglés —algunos países requieren rendir exámenes internacionales o tener conocimiento básico del idioma del país anfitrión-. 
- Contar con un promedio de notas superior a 5.0 en cada una de las asignaturas cursadas durante los dos últimos años.

\section{MARCO TEÓRICO}

\section{a) Multiculturalismo e interculturalidad}

En los últimas décadas diversos países se han definido como multiculturales o interculturales, tales como, Australia, Canadá, Bolivia, Colombia, México, Ecuador, etcétera (Aguilar et al., 2011). En paralelo a la dimensión normativo-constitucional, se ha desarrollado una teoría filosófico-política que promueve la tolerancia y coexistencia de diversas culturas en un mismo territorio, denominada multiculturalismo. Para quienes promueven el pluralismo cultural, la diversidad no debe ser vista como una amenaza; por el contrario, hay que respetarla, aprovecharla y celebrarla. A partir de los valores de igualdad y autonomía, Kymlicka (1996) argumenta a favor de las demandas de minorías culturales que buscan ser reconocidas políticamente en sociedades plurales. Esta línea de pensamiento ha sido desarrollada por los denominados «comunitaristas», quienes han formulado una teoría del reconocimiento que ha sido de gran utilidad para prevenir o resolver conflictos entre distintas culturas. Según Bouchard y Taylor, la interculturalidad es una política o un modelo que propicia relaciones armoniosas entre distintas culturas que, sin eliminar sus diferencias, contribuyen a la integración social y preservación de vínculos sociales (2008:19,118). La interculturalidad «sugiere un proceso activo y permanente de negociación e interrelación donde lo propio y particular no pierdan su diferencia, sino que tengan la oportunidad y capacidad para aportar desde esta diferencia a la creación de nuevas comprensiones, convivencias, colaboraciones y solidaridades» (Walsh, 2008:141). La principal contribución de esta corriente está en el énfasis que hace por la interacción entre sujetos que pertenecen a identidades culturalmente diferentes (Giménez Romero, 2003). En ese sentido, puede afirmarse que el multiculturalismo y la interculturalidad se inscriben dentro pluralismo y se oponen a visiones asimilacionistas y etnocentristas. Lamentablemente, en Chile las experiencias de reconocimiento de la diversidad cultural juvenil no han problematizado en forma adecuada las dimensiones subjetivas de todos los tipos de jóvenes que es posible apreciar en el país (Sandoval, 2003:13). 
El término multiculturalismo suele usarse para describir a una sociedad con características de pluralidad cultural, pero también para aludir a un modelo de política promotora de esa diversidad (Raz, 1994). En este último sentido, cabe mencionar que en las últimas décadas se han diseñado, implementado y evaluado diversas políticas públicas multiculturales en todos los continentes. La principal finalidad que tales políticas persiguen es contribuir a una convivencia más armónica e inclusiva entre distintos grupos culturales que cohabitan en un mismo territorio (Anchustegui Igartua, 2011). Esto se aprecia en programas públicos o estatales que propician la educación intercultural, la participación y representación política de las distintas culturas, la salud intercultural, la resolución judicial y extra judicial de conflictos, el respeto de la lengua y las costumbres de las distintas identidades culturales, etcétera. Si tales políticas y planes son exitosas e inciden en la socialización de los sujetos, es posible avanzar hacia un modelo de ciudadanía multicultural. Para alcanzar mayores niveles de tolerancia y respeto recíproco entre distintas culturas que comparten un mismo territorio se requiere que esas culturas estén dispuestas a dialogar e interactuar. No basta con reconocer una igualdad de derechos entre las distintas culturas, pues se requiere de comunicación y aprendizaje continuo entre miembros de distintas culturas; es decir, un ethos multicultural (Ramón, 2009). Sin embargo, si los resultados de los planes y políticas públicas no son los esperados, es entendible que surjan voces críticas que cuestionen las externalidades del multiculturalismo (Bissoondath, 1994; Blainey, 1984; Gunew, 2004; Maglesini y Giménez, 2000).

Por sensibilidad intercultural se entiende «la habilidad para desarrollar un sentimiento positivo hacia la apreciación y el entendimiento de las diferencias culturales que promueve un comportamiento positivo y efectivo en la comunicación intercultural» (Chen, 1997:10). Bennet ha propuesto un Modelo de Desarrollo de Sensibilidad Intercultural (DMIS) para explicar por qué algunas personas adquieren habilidades para intervenir en contextos interculturales (1993). El DMIS parte del supuesto que el contacto con otras culturas debería generar presión para llevar a cabo un cambio en la propia visión del mundo (Bennet, 2004). Asumiendo que las relaciones entre culturas son necesarias, es interesante investigar por qué algunas personas desarrollan más competencias interculturales que otras. El DMIS distingue seis etapas: negación, defensa, minimización, aceptación, adaptación e integración. Las tres primeras pueden ser agrupadas dentro de la categoría de etnocentristas, mientras que las tres últimas como etnorelativas. Por etnocentrismo se alude a 
«la tendencia a considerar superior la propia cultura y a aplicar los propios valores culturales para juzgar el comportamiento y las creencias de personas criadas en otras culturas» (Kottak, 2002:51). En consecuencia, en las etapas etnocentristas el sujeto percibe a su propia cultura como la central, sin cuestionar las creencias y comportamientos que ha recibido en su socialización temprana. Por el contrario, el relativismo cultural propugna que «el comportamiento de una cultura particular no debe ser juzgado con los patrones de otra cultura» (ibid.). Las fases etnorelativas se caracterizan por aceptar que las creencias y conductas propias son solo una forma más en que el sujeto organiza la realidad, entre tantas otras alternativas. Una de estas sub etapas es la adaptación, en la cual el sujeto no trata de sustituir valores propios sino que incluye comportamientos y creencias nuevas al repertorio propio. La persona modifica su comportamiento para adaptarse al entorno cultural del momento, sin renunciar a los valores propios.

\section{b) Adaptación y readaptación cultural}

El proceso de adaptación cultural implica en el sujeto la confrontación entre la cultura propia con otra cultura que se está conociendo (Maeireizo Tokeshi, 2007). Si la persona trata de integrar elementos de ambas culturas, con tal de adaptarse a la sociedad de acogida, entonces ajusta sus creencias y comportamientos a este nuevo contexto (Berry, 1997). Es importante tener en cuenta que el proceso puede ser aún más difícil por el hecho de no conocer el idioma. Por adaptación sociocultural se entiende la habilidad para lidiar con problemas y asuntos cotidianos, especialmente relacionados con la familia, la escuela y el trabajo (ibid.). El estudiante de intercambio debe enfrentar una nueva realidad social, donde algunos comportamientos y valores que son legítimos en su país de origen puede que no lo sean en el país anfitrión. Por tanto, la mayoría de los estudiantes de intercambio experimentan procesos de cambio y adaptación (Ward, Bochner y Furnham, 2001). Es normal que en dicho proceso surjan cuestionamientos sobre lo aprendido y vivenciado previamente, en especial si en la sociedad de acogida se requiere de ajustes para interactuar en forma adecuada a las nuevas circunstancias (Benatuil y Laurito, 2008).

Diversos factores inciden en la adaptación social, tales como, el sexo, la edad, el estado civil, la distancia cultural, la experiencia internacional previa, etcétera. (Bochner, 1972; Chataway y Berry, 1989; Hull, 1978; Ward y Searle, 1991). Oberg (1960) describe el proceso 
de adaptación de expatriados en cuatro etapas. La primera es denominada «luna de miel» y se estima que su duración promedio es de tres meses. En esta fase existe un sentimiento de entusiasmo y excitación por todo lo nuevo en el país anfitrión. La segunda etapa es conocida como «shock cultural» y en esta etapa lo que parecía ser novedoso y fantástico, se torna habitual e incluso en ocasiones molesto. El shock cultural aumenta por la ansiedad de estar lejos de todo lo familiar y el constante contacto con un nuevo ambiente. Según Oberg, «el shock cultural está precipitado por la ansiedad que resulta de la pérdida de todos los signos familiares y símbolos de interacción social» (1969). El shock se manifiesta en un estado de confusión, frustración y desorientación que experimentan las personas al dejar su cultura (Benatuil y Laurito, 2008). Los estudiantes suelen experimentar el shock cultural con sentimientos de ansiedad, indignación, incomprensión o soledad. La tercera etapa es la «recuperación» donde el individuo es capaz de lidiar con sus emociones y generar una actitud positiva hacia el entorno del país receptor. En esta etapa se aprecia que hay un mayor dominio del idioma adquirido, lo que es beneficioso en términos socio-culturales pues el sujeto está en mejores condiciones para entender aspectos de la cultura extranjera. Por último, en la «adaptación» todo lo que se percibía como extranjero se disfruta. El individuo se acostumbra al país anfitrión, a su gente y cultura.

El estudiante, una vez que concluye su experiencia de intercambio, debe retornar a su país de origen. Un aspecto interesante es que la identidad cultural del sujeto se ha visto modificada, toda vez que al regresar al país de origen el individuo trae consigo aspectos de la cultura anfitriona (Koester, 1984). Dado que la persona ha incorporado elementos culturales foráneos y diferentes a los de la sociedad a la que pertenece, es relevante analizar el proceso de reinserción social (Gaw, 2000). La adaptación que tiene lugar luego del intercambio es también conocida como readaptación. Readaptarse al país de origen toma tiempo, según cada caso en particular, por lo que bien puede decirse que el sujeto se encuentra nuevamente en una etapa de transición (Martin, 1986; Storti, 2001). El shock cultural ocurre tanto en el país anfitrión como en el país de origen, y se refiere a todas las dificultades sociales, psicológicas o emocionales al adaptarse a una nueva cultura o dificultades similares al adaptarse a la cultura propia como resultado de cambios sociales o culturales. Al shock cultural que ocurre en el proceso de reinserción a la cultura de origen se le denomina «shock cultural reverso» (por sus siglas, SCR) (Gaw, 2000). El SCR comenzó a ser estudiado a partir de 
1944, cuando Scheutz examinó las dificultades que enfrentaban los veteranos de las fuerzas armadas al regresar a sus países desde el campo de batalla en la Segunda Guerra Mundial (1944).

La principal diferencia entre shock cultural y SCR proviene de las expectativas del estudiante de intercambio. Muchas veces los estudiantes esperan regresar a un hogar que no ha sufrido cambios durante su ausencia, situación poco habitual (Gaw, 2000). Mientras que algunos estudiantes pueden experimentar pocas dificultades en su readaptación, en otros casos el proceso puede tomar meses e incluso años. Algunos de los problemas más conocidos son: dificultades académicas, conflictos de identidad cultural, depresión, ansiedad y dificultades interpersonales (Martin, 1986; Raschio, 1987; Sahin, 1990). El estudiante se adapta al país de origen cuando se acostumbra a estar en su hogar y no siente el constante deseo de volver al país anfitrión (Storti, 2001). Para dar cuenta de los procesos de adaptación y readaptación, Gullahorn y Gullahorn (1963) reformulan la propuesta de Oberg y sugieren la figura de la «Curva $\mathrm{W} »$, que muestra todas las etapas por la que pasa la persona que realiza un intercambio: i) luna de miel, ii) shock cultural, iii) recuperación, iv) ajuste, v) despedida, vi) SCR, vii) recuperación y viii) ajuste.

FIGURA 1: CURVA W

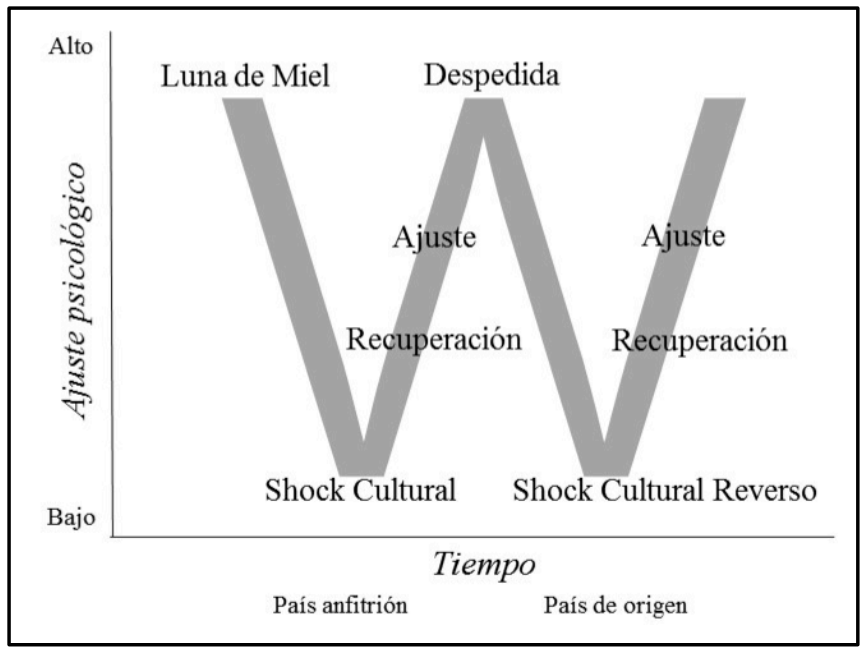

Fuente: elaborado a partir de Oberg (1960) y Gullahorn y Gullahorn (1963). 


\section{MetodologíA}

Para comprender cómo los chilenos evalúan la experiencia de intercambio que vivieron en su adolescencia y algunos de sus efectos en el largo plazo, se ha optado por realizar una investigación social mixta, combinando el análisis de entrevistas en profundidad con la aplicación de una encuesta a los mismos entrevistados. Dado que se trata de un estudio exploratorio, se ha privilegiado la densidad, consistencia y robustez de la interpretación de los hallazgos por sobre el análisis de un número mayor de casos. En ese sentido, se ha preferido llevar a cabo un análisis en profundidad de las percepciones de los participantes en los programas de intercambio. En total fueron entrevistadas seis personas que participaron en programas de intercambio de YFU.

Para identificar a los entrevistados se recurrió a la técnica «bola de nieve» (Noy, 2008), aprovechando la colaboración de informantes clave en YFU Chile. Cada uno de los participantes fue entrevistado en una sesión de dos horas de duración, las que se efectuaron durante los meses de mayo y junio de 2014. A todos los entrevistados se les explicó el sentido de la investigación y se les pidió que firmaran un documento en el que otorgaban su consentimiento para que sus comentarios fuesen grabados y luego interpretados. Se les aseguró confidencialidad a todos los entrevistados y también se les dio la oportunidad de abandonar el proyecto en cualquier momento. Para evitar sesgos, nos aseguramos que los entrevistados no tuviesen ningún vínculo laboral con YFU Chile ni fuesen voluntarios de dicha organización. Es más, si en algún momento fueron voluntarios o realizaron actividades en YFU Chile, ello aconteció hace más de cinco años. Para la definición del cuestionario guía, se efectuó un plan piloto en el mes de abril de 2014, donde fueron entrevistadas otras tres personas. A partir del plan piloto se llegó a la conclusión que era preferible construir un formato de entrevista semi-abierta. Las entrevistas se estructuraron en torno a tres grandes bloques conversacionales:

- Antecedentes sobre el contexto familiar, personal y motivaciones previas a la partida.

- Información sobre la experiencia de intercambio.

- El proceso de readaptación al país y vida posterior.

Los datos recogidos en las entrevistas fueron transcritos e interpreta- 
dos a partir de las recomendaciones metodológicas de Grounded Theory o Teoría Fundamentada en los Datos (Strauss y Corbin, 1994). Las conversaciones fueron codificadas, y así fue posible reunir temas comunes y construir las distintas categorías de análisis. En el mes de julio de 2015 se tomó contacto nuevamente con los entrevistados y se les solicitó que respondieran una breve encuesta sobre el impacto de la experiencia en sus vidas.

Se optó por distinguir tres grupos etarios, pues ello facilitaría la comparación entre personas que se encuentran en distintas etapas de la vida. De esa manera, se entrevistaron dos personas de 25 a 40 años, dos personas de 40 a 55 años, y otras dos personas de más de 55 años. Se quiso mantener igualdad de género entre los entrevistados: tres hombres y tres mujeres. En cuanto a la ciudad de origen, tres provenían de Santiago y los otros tres de regiones (Antofagasta, La Serena y Temuco). Al momento de viajar, cinco estudiaban en colegios particulares y solo uno asistía a un liceo público. Coincidentemente, cinco declaran que al momento del viaje sus familias pertenecían a los sectores económicos acomodados de la sociedad chilena ( $\mathrm{B}, \mathrm{C} 1 \mathrm{a}$ y $\mathrm{C} 1 \mathrm{~b})$, mientras que uno solo pertenecía a una familia de clase media (C2). Respecto del país anfitrión, cinco viajaron a Estados Unidos (estados de Kansas, Michigan y West Virginia) y uno a Nueva Zelanda. Cuatro personas viajaron al extranjero durante un año, mientras que los otros dos lo hicieron por seis meses. En la actualidad los seis ex participantes de programas de YFU Chile residen en Santiago. En cuanto a sus actividades, la mitad son empresarios y la otra mitad son profesionales que estudiaron derecho, veterinaria o trabajo social. Dos declaran en estos momentos formar parte del grupo socioeconómico C1a (i.e. con ingresos entre 2.8 y 6 millones de pesos mensuales), tres pertenecen al nivel $\mathrm{C} 1 \mathrm{~b}$ (i.e. con ingresos mensuales entre 1.5 y 2.8 millones de pesos) y uno considera que se encuentra en el nivel C2 (i.e. con ingresos mensuales entre 900 mil y 1.5 millones de pesos). Es interesante constatar que uno solo declara que sus ingresos actuales son superiores a los ingresos de su familia al momento de viajar, uno indica que no ha habido variaciones en términos de nivel socioeconómico al que se pertenece, mientras que los otros cuatro estiman que los ingresos de sus hogares actuales son inferiores a los ingresos que tenían sus familias al momento de viajar. Al consultárseles si la experiencia de intercambio estudiantil les permitió o no ascender socialmente, cuatro indicaron que no, mientras que dos sostienen que existe una relación causal entre el intercambio y su ascenso en la escala social. 


\section{CUADRO 1: ANTECEDENTES SOCIODEMOGRÁFICOS \\ DE LAS PERSONAS ENTREVISTADAS}

\begin{tabular}{|c|c|c|c|c|c|c|}
\hline $\begin{array}{c}\text { Código de } \\
\text { entrevista }\end{array}$ & I & II & III & IV & V & VI \\
\hline Edad actual & 34 & 33 & 45 & 52 & 59 & 66 \\
\hline $\begin{array}{c}\text { Año de } \\
\text { intercambio }\end{array}$ & $1997-1998$ & $\begin{array}{c}1997- \\
1998\end{array}$ & $\begin{array}{c}1984- \\
1985\end{array}$ & $1978-1979$ & $1970-1971$ & $1965-1966$ \\
\hline Sexo & Mujer & Hombre & Mujer & Hombre & Mujer & Hombre \\
\hline $\begin{array}{c}\text { Tipo de } \\
\text { Colegio }\end{array}$ & Particular & Particular & Particular & Particular & Particular & Público \\
\hline $\begin{array}{c}\text { NSE al viajar } \\
\text { NSE actual }\end{array}$ & C1a 2 & B & C1b & C2 & B & C1a \\
\hline $\begin{array}{c}\text { Variación } \\
\text { NSE }\end{array}$ & $\downarrow$ & $\downarrow$ & $=$ & $\uparrow$ & $\downarrow$ & $\downarrow$ \\
\hline $\begin{array}{c}\text { Ascenso } \\
\text { social }\end{array}$ & No & No & Sí & No & No & Sí \\
\hline
\end{tabular}

Fuente: elaboración propia (las personas entrevistadas son identificadas con números romanos en cursiva; en números arábigos se identifica el párrafo respectivo de la entrevista).

\section{PRinCipales Resultados}

En esta sección se presentan los resultados de las entrevistas, ordenados cronológica y temáticamente de la siguiente manera: a) Antecedentes anteriores a la experiencia del intercambio: motivaciones, sentimientos y expectativas; b) Información sobre la experiencia de intercambio: rol de la familia anfitriona, adaptación y dificultades, y c) Readaptación y vida posterior: readaptación, valoración, cambios atribuidos a la experiencia de intercambio y proyección futura.

\section{a) antecedentes anteriores a la experiencia de intercambio}

Las personas entrevistadas compartieron distintas motivaciones por las cuales optaron por participar en el programa de intercambio. Entre las motivaciones internas se encuentran el deseo del entrevistado por querer tener una experiencia de intercambio en otro país. En las entrevistas se mencionaron diversos intereses, tales como, conocer otras 
culturas, aprender otro idioma, salir de la rutina y la oportunidad de descubrir realidades nuevas (II 10, III 15, IV 4, V 8). A su vez, los entrevistados vieron que podía tratarse de una oportunidad para madurar, crecer, independizarse y adquirir herramientas para su posterior desarrollo personal y profesional. Además de las inquietudes personales, también influyeron factores externos en la decisión, tales como, la experiencia de algún familiar o amistad cercana que participó previamente en la experiencia de intercambio en el extranjero y que compartió su historia con los entrevistados. Los padres y el colegio influyeron en algunos de los entrevistados en la toma de la decisión de participar en un programa como estudiante de intercambio (I 8, II 2, III 2, III 19, IV 2, V 2, VI 2).

En el colegio siempre hubo gente que de repente se iba de intercambio y se desaparecía... Entonces, siempre estuvo el bichito o la duda de cómo sería (I 8).

Mi hermana viajó antes que yo y tuvo una experiencia muy buena, una familia muy buena. Yo soy la menor de tres y siempre me sentí súper protegida. Pensaba que este viaje me iba a hacer súper bien porque iba a madurar y crecer (V 2).

Los principales sentimientos nombrados por los entrevistados al describir su situación previa al intercambio son los siguientes: miedo, indecisión, ansiedad, euforia, felicidad, excitación e incertidumbre (I 45, III 25, III 27, IV 22). Dentro de las expectativas los entrevistados mencionan: aprender el idioma, conocer gente, involucrarse en otra cultura, expectativas de autonomía (III 21, IV 4, V 8).

Consultados sobre su vida familiar antes de viajar al extranjero, la mayoría de los entrevistados explicó que vivía con sus padres, que no tenían más responsabilidades que estudiar y participar en actividades extra programáticas como hacer deporte. Sentían que vivían en un ambiente protegido y privilegiado, sin mayores preocupaciones (I 6, II 8, III 11, IV 16, V 4). Dado que contaban con servicio doméstico, por lo general no colaboraban en el hogar en tareas de limpieza, cocina, etcétera. Solo uno de los participantes se mostró autocrítico con su forma de ser antes del intercambio:

La verdad es que yo vivía en una burbuja, hijita de papá (I 8). Yo no hacía nada [en la casa]. Me levantaba y dejaba todo tirado, volvía del colegio y estaba todo limpio, el almuerzo hecho, no había que hacer nada (I 61). 


\section{b) Información sobre la experiencia de intercambio}

Uno de los pilares fundamentales de la experiencia de intercambio es la familia anfitriona. La familia anfitriona es quien, de manera voluntaria, decide hospedar al estudiante durante un año, ser su guía y compartir su vida. En general los entrevistados mencionaron haber tenido una buena relación con la familia anfitriona (I 55, II 38, III 43, IV 32), y en muchos casos han mantenido contacto a pesar del paso de los años. Sin embargo, uno de los entrevistados mencionó no haber tenido una buena relación durante el intercambio, de modo que hace años que no mantiene contacto con las personas que conoció en el país anfitrión (V 35). Al preguntárseles sobre cómo describirían a las familias que los recibieron, indicaron que habían sido integradoras, agradables, cariñosas y cálidas (I 47, II 94, III 29, VI 10).

Conocí tanta gente buena. Tanta gente valiosa, esa familia que no tenía por qué tratarme como me trató, increíble, impresionante, nunca me sentí mal (II 94).

Fue una familia agradable, cariñosa, bien integradora; la verdad me integraron y nunca tuve un problema [...] Yo tenía todas las condiciones, una ciudad bonita, colegio bonito, familia agradable. No eran ni muy top ni nada, todo en su punto medio (III 29).

Durante los primeros meses; es decir, en el período de adaptación, los estudiantes se enfrentan a situaciones nuevas constantemente y deben hacerse cargo por sí mismos de los problemas que tengan. Por el hecho de estar geográficamente distanciados de su círculo íntimo, deben explorar alternativas para enfrentar las adversidades o situaciones incómodas, incluyendo la búsqueda de nuevos apoyos, consejos o acompañamiento. Los ex alumnos entrevistados reconocen que los primeros tres meses son el período más problemático del intercambio (I 116, II 34, V 41). Las dificultades tienen que ver principalmente con el hecho de sentirse solo, no conocer el idioma, y también a diferencias culturales a las cuales deben acostumbrarse (II 32, IV 30, V 24 , VI 20). Este proceso muchas veces provoca que los estudiantes quieran abandonar la experiencia y volver a lo conocido o retornar a su zona de confort (I 114, V 59). En estos casos es clave el apoyo que reciban por parte de sus familias anfitrionas, amigos y la organización que patrocina el intercambio. 
Yo te diría que el mes que más me costó fue el tercero. Como ya pasó el mes de vacaciones, ahí quería mi casa, quiero mi comida, quiero mi ropa, quiero mi cama, quiero mis cosas (I 114).

\section{c) Readaptación y vida posterior}

En esta sección se presentan las valoraciones que hicieron los entrevistados de sus experiencias de intercambio, es decir, del significado que ellos otorgan al año que estuvieron viviendo en el extranjero, los cambios atribuidos al intercambio y la posterior vinculación de la experiencia en diversos ámbitos de la vida: profesional, personal y social.

i) Proceso de readaptación. La percepción de los entrevistados sobre la readaptación es ambigua. Algunos mencionan haber tenido dificultades interpersonales y académicas, haber extrañado su vida previa en el extranjero e incluso haber estado con depresión (I 181, II 96, III 74, IV 59). Algunos ex alumnos incluso manifestaron haber tenido una valoración peyorativa de su propia cultura al momento de regresar al país. Esto pudo haber incidido en la decisión de algunos de haber cursado estudios de pregrado en el extranjero (I 108, V 45). Otros, sin embargo, mencionan no haber tenido dificultades durante el proceso de readaptación (V 61, VI 101).

Eso para mí fue súper duro, volver y como saber que no ibas a poder seguir esa relación [de pololeo] (III 74).

En ese minuto yo quería más a mi familia [anfitriona en el país extranjero] que a mi propia familia [biológica] (II 90).

Cabe destacar que se puede apreciar una relación directa entre una experiencia satisfactoria con generación de lazos y dificultades de readaptación. Aquellos entrevistados que manifestaron no tener dificultades durante la readaptación, no hicieron énfasis en los vínculos generados durante la experiencia con la familia y amigos.

ii) Valoración general por parte de los estudiantes. Todos los entrevistados valoraron a la experiencia de intercambio en general como positiva (I 146, II 2, III 87, IV 77, V 4, VI 99). Sin embargo, lo que más se valoró fue el haber adquirido herramientas útiles como otro idioma, los aprendizajes de otras culturas y las amistades generadas. 
Por otro lado, ciertos aspectos de la cultura anfitriona fueron valorados en forma negativa, por ejemplo, cinismo, ignorancia, discriminación y racismo (III 87, VI 79). Cabe mencionar que uno de los entrevistados no quedó conforme con la experiencia, sin embargo, la evalúa como un aprendizaje importante en su vida (V 65).

Fue la mejor experiencia de mi vida, hay un antes y un después, segurísimo (II 2). El intercambio es lo mejor que me ha pasado en la vida (II 92).

El intercambio significó un antes y un después en mi vida, un cambio de rumbo radical, una experiencia de vida [...] Esa inquietud de seguir conociendo, seguir conociendo otras culturas. Integrarse a otro grupo, con personas totalmente diferentes, de otras culturas. Para mí eso es el intercambio, una experiencia muy enriquecedora en todos los sentidos. En un mundo globalizado como el que vivimos el que tiene esa experiencia tiene más sensibilidad con lo que pasa y puede enfrentar los problemas. El intercambio ha ayudado en los temas profesionales a tomarlos de otra manera, porque conocí otras realidades (II 128).

Yo creo que todo lo que me tocó fue bueno, la familia, la ciudad, el entorno, las amigas, todo fue bueno (III 87).

Esas percepciones fueron coincidentes con las respuestas que los participantes proporcionaron un año después de las entrevistas, al contestar una encuesta sobre el impacto de la experiencia de intercambio en sus vidas. La valoración del intercambio respecto de su desarrollo profesional actual es altísima. Cinco de seis le atribuyen al intercambio importancia en cuanto a su desempeño en la universidad y competitividad en el mercado laboral. En cambio, las opiniones son más variadas respecto del impacto de la experiencia de intercambio en los resultados obtenidos en la Prueba de Aptitud Académica (PAA) o en la Prueba de Selección Universitaria (PSU) y en cuanto a su nivel actual de ingresos. La tabla que sigue muestra los principales resultados a propósito de la escala Likert aplicada en el cuestionario: «En una escala de 1 a 7 , siendo 1 «nada» y 7 «mucho», por favor señale su nivel de conformidad con las siguientes afirmaciones sobre su experiencia de intercambio estudiantil». 


\section{CUADRO 2: EVALUACIÓN DE LA EXPERIENCIA DE INTERCAMBIO Y DESARROLLO ACADÉMICO-PROFESIONAL}

\begin{tabular}{|l|c|c|c|c|c|c|c|}
\hline Variable & I & II & III & IV & V & VI & Promedio \\
\hline $\begin{array}{l}\text { Me ha servido en mi desarrollo } \\
\text { profesional. }\end{array}$ & 7 & 7 & 7 & 6 & 7 & 7 & 6,8 \\
\hline $\begin{array}{l}\text { Me ayudó a lograr un buen } \\
\text { desempeño en la universidad. }\end{array}$ & 6 & 6 & 6 & 6 & 1 & 5 & 5,0 \\
\hline $\begin{array}{l}\text { Me ayudó a obtener un buen puntaje } \\
\text { en la Prueba de Aptitud Académica o } \\
\text { Prueba de Selección Universitaria. }\end{array}$ & NA & 1 & 5 & 3 & 1 & 4 & 2,8 \\
\hline $\begin{array}{l}\text { Ha fortalecido mi competitividad en el } \\
\text { mercado laboral. }\end{array}$ & 4 & 7 & 7 & 6 & 1 & 5 & 5,0 \\
\hline $\begin{array}{l}\text { Si no hubiera sido por la experiencia } \\
\text { de intercambio, mis } \\
\text { ingresos actuales serían menores. }\end{array}$ & 4 & 6 & 5 & 5 & 1 & 5 & 4,3 \\
\hline
\end{tabular}

Fuente: elaboración propia.

Al ser consultados sobre el impacto socioeconómico que tuvo la experiencia de intercambio en sus vidas, las respuestas también fueron variadas:

\section{CUADRO 3: IMPACTO SOCIOECONÓMICO DE LA EXPERIENCIA DE INTERCAMBIO}

\begin{tabular}{|c|l|}
\hline I & $\begin{array}{l}\text { Debido a que cuando me fui de intercambio quienes me mantenían eran mis } \\
\text { padres y ellos mantienen su situación económica o estrato socioeconómico, no } \\
\text { creo que el intercambio haya influido de manera directa en mi estado actual. } \\
\text { Creo que va más relacionado a la carrera que estudié y a las decisiones que he } \\
\text { tomado en cuanto a darle prioridad a mi desarrollo personal por sobre mi situa- } \\
\text { ción económica. [...] }\end{array}$ \\
\hline III & $\begin{array}{l}\text { Lo más destacable son las habilidades adaptativas que se requieren desarrollar, } \\
\text { las que hoy son fundamentales en el campo laboral. }\end{array}$ \\
\hline IV & $\begin{array}{l}\text { Aprender inglés en profundidad [...] ha tenido una gran influencia en mi desa- } \\
\text { rrollo profesional. Trabajo [...] con varias empresas internacionales (proveedo- } \\
\text { res y clientes), donde esta ventaja es notable. Por otra parte, la experiencia de } \\
\text { tratar con personas de diversas culturas y nacionalidades (norteamericanos, en } \\
\text { el caso de la familia que me tocó, y estudiantes de intercambio de muchos otros } \\
\text { países) sin duda me ha ayudado a tener, valorar y acrecentar una gran variedad } \\
\text { de relaciones en todo el mundo, tanto personales como profesionales. }\end{array}$ \\
\hline V & $\begin{array}{l}\text { Más que un impacto socioeconómico, para mí fue uno de naturaleza cultural. } \\
\text { Vivir en medio de una familia ausente y desavenida me hizo madurar frente a } \\
\text { las duras realidades de la vida. El impacto en lo económico, vivir la experiencia } \\
\text { de una familia con menores recursos y limitaciones, siendo la única sostenedora } \\
\text { del hogar una mujer viuda. Quedaba poco tiempo y energía para hacer familia. } \\
\text { No fue una situación agradable y ciertamente no mi ideal de vida; sin embargo, } \\
\text { fue una experiencia muy interesante. }\end{array}$ \\
\hline VI & $\begin{array}{l}\text { El hecho de haber estado en Estados Unidos no produjo un impacto socio } \\
\text { económico en mi persona, debido a que después entré a trabajar con mi padre, } \\
\text { siguiendo una tradición familiar. }\end{array}$ \\
\hline
\end{tabular}


Fuente: elaboración propia (a partir de pregunta abierta en encuesta aplicada a los entrevistados).

iii) Cambios atribuidos al intercambio. Indudablemente, la experiencia de vivir durante un año en una cultura ajena a la propia genera cambios en una persona que se encuentra en etapa de formación. Los entrevistados destacaron diversos cambios positivos: madurez, independencia y desarrollo de opinión propia (I 146, II 40, III 60, V 4, VI 73); mayor interés por lo internacional (II 80, II 122); valoración de la diversidad cultural y disminución de prejuicios (II 128, II 130, IV 95); tolerancia y respeto de otros puntos de vista (I 221, II 130, I 146); interés por lo social (IV 131, VI 103), y desarrollo de habilidades de interacción y comunicación (II 90, III 84),

Cuando me fui era un niño y cuando volví era un hombre que podía resolver problemas solo, sin preguntar nada a nadie (II 88). Es como una adicción, para mí eso es intercambio, esa búsqueda incesante de conocer otras culturas, otros idiomas, de viajar, integrarse a otros grupos, con personas totalmente diferentes y de otras culturas (II 128).

Es algo como inolvidable, que te ayuda a madurar, que sirve de muchas formas. No solo en la parte intelectual se aprende, además la experiencia te enseña a valorar las cosas (I 221). Te sirve mucho, te ayuda a madurar y ver las cosas de otras perspectivas (I 146).

Y mi personalidad ha cambiado, en todo sentido, la manera de enfrentar los problemas, mi personalidad, este tema de haber sido introvertido, etcétera (VI 90). Algo lindo que hay en la vida es viajar. Uno se siente diferente, intercambia con la gente, la cultura, conocer los países vecinos (VI 103).

iv) Proyección de la experiencia de intercambio en la vida futura. Todos los entrevistados mencionaron distintos aspectos obtenidos durante el intercambio y que han contribuido de manera sustantiva en cómo han sido sus vidas. Esto se aprecia en distintos planos de la vida, tanto en el nivel personal como profesional y social. Lo más reiterado por los entrevistados fue la utilidad que ha tenido en sus vidas el haber adquirido un segundo idioma, tanto en lo profesional como en el ámbito social (I 203, II 108, III 76, IV 101, VI 60). Otro de los temas mencionados fue la continuidad de los vínculos de amistad y confianza generados con sus amigos y la familia anfitriona (I 197, II 38, VI 44), los que gracias a internet se han reforzado (v. gr., Facebook, Skype, email, etcétera.). Incluso la mitad de los entrevistados formaron pareja personas de nacionalidad extranjera (II, III, IV). Otro aspecto que vale 
la pena ser destacado es el interés que algunos entrevistados dicen haber desarrollado por las temáticas globales e internacionales (II 80, III 105, V 77). Por ejemplo, dos de los entrevistados continuaron sus estudios superiores en el exterior, y uno de ellos está planeando en la actualidad cursar estudios de postgrado en el extranjero (I 108, II 80, V 45).

\section{Discusión}

Las personas entrevistadas que participaron en los programas de YFU señalan que la experiencia de intercambio implicó cambios sustantivos en su forma de ser y comprender el mundo. En general ellos están conscientes que sus propias identidades cambiaron luego del intercambio, expandiendo su horizonte de expectativas y significados. Los entrevistados advierten en sus personalidades diferencias relevantes en comparación con sus pares y familiares que no han tenido la posibilidad de vivir durante un período prolongado de tiempo en el extranjero. También se dan cuenta que han evaluado y asimilado algunos aspectos de la cultura anfitriona que valoran y comparten, integrándolas con su idiosincrasia o cultura de origen. Ese ejercicio requiere la voluntad de conocer otras culturas y de estar dispuesto al intercambio cultural, en el entendido que la propia identidad del sujeto puede verse modificada si se apropia de códigos o patrones culturales que le parecen relevantes o le hacen sentido en su vida cotidiana. Al mismo tiempo, los estudiantes que participan en programas de intercambio requieren de una actitud de humildad al regresar, en especial cuando los demás quieren conocer las experiencias que vivieron. Uno de los entrevistados comentó que al retornar al país adoptó posturas arrogantes, como queriendo hacerle ver a sus pares que no conocían el mundo o que tenían actitudes tercermundistas (II 96).

Otro asunto no menor es la capacidad que adquieren los participantes en programas de intercambio de adaptarse a nuevos contextos o circunstancias, incluso si son adversas. El hecho de haber abandonado por un tiempo la zona de confort que se tenía durante la adolescencia, quienes participan en programas de intercambio suelen desarrollar capacidades adaptativas superiores a las personas que no han experimentado cambios radicales en sus vidas. Sumado a lo anterior, pareciera ser que las personas que participan en este tipo de programas son menos temerosas a los cambios en las distintas dimensiones de la vida (personal, familiar, social, profesional). Más que temerle al cambio, estos programas aumentan el interés y curiosidad de los participantes 
por conocer y explorar nuevas experiencias y situaciones. Como lo ratificara uno de los entrevistados: «lo que detonó este tema de no querer parar de conocer nunca fue el intercambio» (II 106).

Un aspecto relevante para esta investigación era identificar si los participantes en los programas de intercambio retornaban al país valorando más que antes la diversidad cultural. A propósito de los comentarios proporcionados por los entrevistados, incluyendo las entrevistas del plan piloto, quisimos complementar los hallazgos preliminares con un sencillo experimento social.

\section{FIGURA 2: AUTORRETRATOS INTERCULTURALES DE VOLUNTARIOS DE YFU CHILE (2014)}

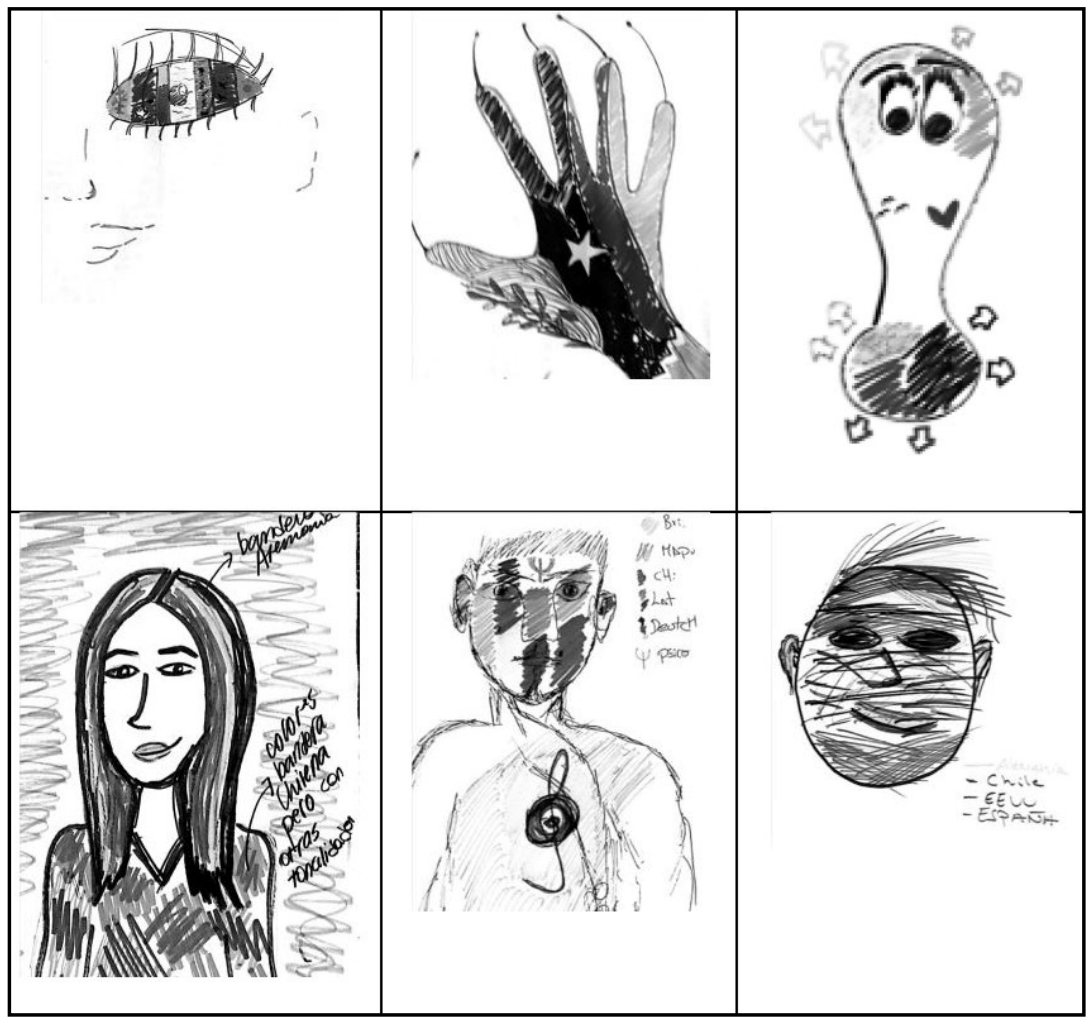

Fuente: elaboración propia a partir de los dibujos de ex participantes en programas de YFU (mayo de 2014). Esta técnica de investigación social ha sido desarrollada por Sarri Vuorisalo-Tiitinen (Universidad de Helsinki), a propósito de sus investigaciones sobre inmigrantes latinoamericanos en Finlandia. 
En una jornada de capacitación de YFU para sus ex alumnos de intercambio que han retornado al país en los últimos cinco años y que desean colaborar con la institución como voluntarios, les pedimos que en quince minutos dibujaran un auto retrato, utilizando un color diferente para cada cultura que ellos consideraran que había influido en su identidad. Fue interesante constatar la magnitud de la interculturalidad que este grupo de voluntarios de YFU considera tener. También llama la atención que algunos ex alumnos destaquen que esa diversidad cultural se encuentra relacionada con algunos de los cinco sentidos del cuerpo humano: vista, olfato, gusto, tacto y audición. A modo de ejemplo, a continuación se reproducen seis de los dibujos confeccionados por los ex alumnos y actuales voluntarios de YFU.

Otro aspecto interesante es la capacidad de autoreflexión y toma de consciencia que adquieren las personas que participan en programas de intercambio. Pareciera ser que son más independientes y más seguros de sí mismos, en el sentido que son menos influenciables en sus decisiones que personas que están fuertemente integradas a una comunidad o sociedad. A la luz de las entrevistas realizadas, es posible concluir que los participantes de programas de intercambio consideran que no solo tienen mayores habilidades de interacción social, sino que además se encuentran mejor preparados para defender sus propias ideas ante los demás. Es interesante que gracias a los programas de intercambio las personas sientan que se ven fortalecidas sus capacidades de argumentación y de generación de pensamiento propio e independiente. En cierto sentido, los estudiantes de intercambio se dan cuenta que son capaces de mirar la realidad en la que se encuentran de una manera crítica, pero a la vez propositiva.

Pero lo anterior también puede tener un correlato negativo. Pareciera ser que las personas que participan en programas de intercambio durante su adolescencia son menos apegadas a sus círculos íntimos, a sus raíces y tradiciones, en comparación con personas que no han tenido la posibilidad de vivir durante un período prolongado de tiempo en el extranjero. Esa capacidad de desanclaje de quienes han participado en programas de intercambio puede ser vista de manera negativa por sus pares y familiares, pues consideran que son personas desapegadas, frías e incluso insensibles.

Tal como lo informara uno de los entrevistados, el intercambio generó en él una capacidad de desapego muy grande, pudiendo trasladarse a vivir a otro país con mucha facilidad (II 118). Recuérdese además que otros dos entrevistados al poco tiempo de haber regresado 
a vivir a Chile optaron por cursar sus estudios universitarios en otro país.

\section{CONCLUSIÓN}

En esta investigación se ha querido mostrar las evaluaciones que - luego de transcurridos varios años e incluso décadas - realizan las personas que han participado en programas de intercambio en el extranjero durante su adolescencia. Las entrevistas realizadas a seis chilenos - de 33 a 66 años de edad - que viajaron al extranjero a través de YFU durante su adolescencia, han permitido conocer en profundidad las motivaciones y expectativas que tenían antes de viajar, los aspectos más relevantes de sus experiencias durante el intercambio, los procesos de adaptación y readaptación cultural que vivieron, el impacto y las externalidades positivas que el intercambio estudiantil ha tenido en sus vidas. La obtención de un segundo idioma y el conocimiento de otra cultura fueron algunos de los aspectos destacados por todos los participantes. Además, quienes han realizado intercambios reconocen que la experiencia les ha otorgado una mayor sensibilidad intercultural e interés por conocer otras culturas. En general la experiencia es evaluada de manera positiva y como una etapa muy significativa en sus vidas. El intercambio no solo les hizo madurar sino que ser más tolerantes y respetuosos con los demás, seguros y confiados de sí mismos, capaces de generar su propio pensamiento propio e independiente. Además, los entrevistados consideran que el intercambio aumentó sus habilidades para adaptarse a distintas circunstancias en la vida, sin temor a los cambios ni a asumir ciertos riesgos, a no conformarse con la zona de confort y a atreverse a explorar nuevas emociones y oportunidades.

SANTIAGO (CHILE), OCTUBRE 2014

RECIBIDO: OCTUBRE 2014 ACEPTADO: JUNIO 2015 


\section{REFERENCIAS BIBLIOGRÁFICAS}

Aguilar, G., S. LAfosse, H. Rojas y R. Steward (2011): Justicia constitucional y modelos de reconocimiento de los pueblos indígenas. México, D.F.: Editorial Porrúa/UNAM.

ANCHUSTEGUi IGARTUA, E. (2011): «Debate en torno al multiculturalismo: Ciudadanía y pluralidad cultural», Araucaria, Revista Iberoamericana de Filosofía, política y humanidades. 13 (26).

BACHNER, D. y U. ZEUTSCHEL (1994): Utilizing the Effects of Youth Exchange: A study of subequent lives of German and American high school exchange participants. New York: Council of International Educational Exchange.

BENATUIL, D. y J. LAURITO (2008): La adaptación cultural en los estudiantes extranjeros. Buenos Aires: Universidad de Palermo.

BennetT, M. J. (2004): «Becoming Interculturally Competent». En J. S. WURZEL (ed.): Toward Multiculturalism: A reader in multicultural education. Newton, MA: Intercultural Resource Corporation.

BERRY, J. W. (1997): «Immigration, acculturation and adaptation». Applied Psychology: An International Review.

BlaineY, G. (1984): All for Australia. North Ryde: Methuen Haynes.

BOCHNER, S. (1972): «Problems in culture learning». En S. BochNER \& P. P. WICKS (eds.): Overseas Students in Australia. Sydney: The New South Wales University Press.

Bouchard, G. y C. TAYlor (2008): Building the Future: A Time for Reconciliation. Quebec. Disponible en: www.accommodements-quebec.ca.

CAstro, G. (2007): «Jóvenes: la identidad social y la construcción de la memoria». Última Década $\mathrm{N}^{\circ} 26$. Valparaíso: Ediciones CIDPA.

CAumont, L. (2014): «El liderazgo de los estudiantes de intercambio uruguayos». Revista Iberoamericana de Ciencias Empresariales y Economía, Año 5, No5.

Chataway, C. J. y J. W. BerRy (1989): «Acculturation experiences, appraisal, coping, and adaptation. A comparison of Hong Kong, Chinese, French and English students in Canada». Canadian Journal of Behavioral Sciences, 21(3).

CHEN, G. M. (1997): «A review of the concept of intercultural sensitivity». Human Communication, 1.

DALY, A. (2002): Factors Impacting Upon Australian University Student Participation in Educational Exchange Programs. New Zealand: Christchurch College of Education.

Detweiler, R. (1984): Final Report of Cross-Sectional Study of 1971 to 1982 Participants and Longitudinal Study of 1983 Participants. Washington: Youth for Understanding.

GAW, K. F. (2000): «Reverse Culture Shock in Students Returning from Overseas». International Journal of Intercultural Relations, 24. 
GIMÉNEZ ROMERO, C. (2003): «Pluralismo, multiculturalismo e interculturalidad». Educación y Futuro: Revista de Investigación Aplicada y Experiencias Educativas $\mathrm{N}^{\circ} 8$.

GullahorN, J. T. y J. E. GULlAHORN (1963): «An Extension of the U-Curve Hypothesis». Journal of Social Issues, 19.

Gunew, S. (2004): Haunted Nations: The colonial dimensions of multiculturalism. London: Routledge.

HAnsel, B. (1986): «The AFS impact study: Final Report». Research Reports, 33 .

Hull, F. (1978): Foreign students in the United States of America: Coping behavior within the educational environment. New York: Praeger.

INTERNATIONALER JUGENDAUSTAUSCH UND BESUCHERDIENST (IJAB) (2005): Long-term effects of the participation in international youth exchange programmes on the participants' personality development. Disponible en línea: www.bkj.de.

Koester, J. (1984): «Communication and the Intercultural Reentry: A Course Proposal». Communication Education, 33.

Kottak, C. P. (2002): Antropología cultural. México, D.F.: McGraw-Hill.

KyMLICKA, W. (1996): Ciudadanía multicultural: una teoría liberal de los derechos de las minorías. Barcelona: Editorial Paidós.

MALGESINI, G. y C. GIMÉNEZ (2000): «Interculturalidad». Guía de conceptos sobre migraciones, racismo e interculturalidad.

MARTIN, J. N. (1986): «Communication in the Intercultural Reentry: Student Sojourners Perceptions of Change in Reentry Relationship» International Journal of Intercultural Relations, 10.

NoY, C. (2008): «Sampling Knowledge: The Hermeneutics of Snowball Sampling in Qualitative Research». International Journal of Social Research Methodology, 11(4).

NunAN, P. (2006): «An Exploration of the Long Term Effects of Student Exchange Experiences». Australian International Education Conference.

OBERG, K. (1960): «Culture Shock: Adjustment to New Cultural Environments». Practical Anthropology, 7.

RAMÓN, G. (2009): «¿Plurinacionalidad o interculturalidad en la Constitución?». En A. Acosta \& E. Martínez (compiladores): Plurinacionalidad: democracia en la diversidad. Quito: Abya Yala.

RASCHIO, R. A. (1987): «College students' perceptions of reverse culture shock and reentry adjustments». Journal of College Student Personnel, 17.

RAZ, J. (1994): Multiculturalism: A Liberal Perspective. Oxford: Clarendon Press.

Reyes, M. I.; J. Rosales, J. Arroyo y A. LeÓn (2013): «Impacto del programa de movilidad estudiantil: bajo la percepción del profesor de la facultad de pedagogía e innovación educativa». Revista Iberoamericana de Producción Académica y Gestión Educativa, Centro de Estudios e Investigación para el Desarrollo Docente. 
Richardson, J. (1981): An American Perspective on Cultural Exchange. Tokyo: Japan Foundation.

SAHIN, N. H. (1990): «Re-entry and the Academic and Psychological Problems of the Second Generation». Psychology and Developing Societies, 2.

SANDOVAL, J. (2003): «Ciudadanía y juventud: el dilema entre la integración social y la diversidad cultural». Última Década $\mathrm{N}^{\circ} 19$. Valparaíso: Ediciones CIDPA.

Schuetz, A. (1944): «The Stranger: An Essay in Social Psychology». American Journal of Sociology, 49(6).

SEPÚlVEDA, L. (2013): «Juventud como transición: elementos conceptuales y perspectivas de investigación en el tiempo actual». Última Década N³9. Valparaíso: Ediciones CIDPA.

StORTI, C. (2001): The Art of Coming Home. Yarmouth, ME: Intercultural Press.

Strauss, A. y J. CORBIN (1990): Basics of Qualitative Research: Grounded Theory Procedures and Techniques. Londres: Sage.

Thomas, A., C. Chang y H. ABT (2006): Outcomes, which make a difference. Long-Term Effects of Participating in International Youth Encounters. Göttingen: Vandenhoeck \& Ruprecht.

Thomas, A. (s. d.): «Which are the Long-Term Personality Effects of Participating in International Youth Exchange?».

Disponible en línea: www.coe.int.

UNESCO (1998): «Declaración mundial sobre la educación superior en el siglo XXI: visión y acción». Disponible en línea: www.unesco.org.

WALSH, C. (2008): «Interculturalidad, plurinacionalidad y decolonialidad: las insurgencias político-epistémicas de refundar el Estado». Tabula Rasa, 9.

WARD, C., S. BOCHnER y A. Furnham (2001): The Psychology of Culture Shock. Philadelphia: Routledge.

- y W. SEARLE (1991): «The Impact of Value Discrepancies and Cultural Identity on Psychological and Sociocultural Adjustment of Sojourners». International Journal of Intercultural Relations, 15(2).

Wilson, A. (1985): «Exchange Students as Bridges between Cultures». Global Education and International Exchange Intercom.

YOUTH FOR UNDERSTANDING: «YFU International Conference 2012. Creating YFU-ture». Arlington, Virginia. 